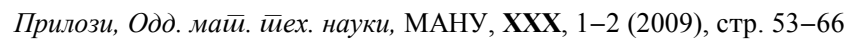
Contributions, Sec. Math. Tech. Sci., MANU, XXX, 1-2 (2009), pp. 53-66

ISSN 0351-3246

UDC: $517.624: 512.643$

Original scientific paper

\title{
EXPONENTIAL CONVEXITY OF THE FAVARD'S INEQUALITY AND RELATED RESULTS*
}

\author{
Naveed Latif, Josip Pečarić, Ivan Perić
}

\begin{abstract}
A b s t r a c t: In this paper we prove positive semi-definiteness of matrices generated by differences deduced from unweighted and weighted Favard's inequality. This implies a surprising property of exponential convexity of this differences which allows us to deduce Gram's, Lyapunov's and Dresher's types of inequalities for this differences.
\end{abstract}

2000 Mathematics Subject Classification. 26A46, 26A48.

Key words: Favard's inequality; weighted Favard's inequality; positive semi-definite matrix; exponential convexity; log-convexity

\section{INTRODUCTION AND PRELIMINARIES}

Let $f$ and $p$ be two positive measurable real valued functions defined on $(a, b) \subseteq \mathbb{R}$ with $\int_{a}^{b} p(x) d x=1$. From theory of convex functions (cf $[1,2,6]$ ), the well-known Jensen's inequality gives that for $t<0$ or $t>1$,

$$
\int_{a}^{b} p(x) f^{t}(x) d x \geq\left(\int_{a}^{b} p(x) f(x) d x\right)^{t}
$$

and reverse inequality holds for $0<t<1$.

${ }^{*}$ This research work is funded by Higher Education Commission Pakistan. The research of the second author and third author are supported by the Croatian Ministry of Science, Education and Sports under the Research Grants 117-1170889-0888 and 058-1170889-1050 respectively. 
Let us write the well-known Favard's inequality:

Theorem 1.1. Let $f$ be a concave non-negative function on $[a, b] \subset \mathbb{R}$. If $q>1$, then

$$
\frac{2^{q}}{q+1}\left(\frac{1}{b-a} \int_{a}^{b} f(x) d x\right)^{q} \geq \frac{1}{b-a} \int_{a}^{b} f^{q}(x) d x
$$

If $0<\mathrm{q}<1$, then the reverse inequality holds in (2).

Let us note that Theorem 1.1 can be obtained from the following result, also obtained by Favard (cf. [7, p. 212]).

Theorem 1.2. Let $f$ be a non-negative continuous concave function on $[a ; b]$, not identically zero, and $\phi$ be a convex function on $[0,2 \tilde{f}]$, were

$$
\tilde{f}=\frac{1}{b-a} \int_{a}^{b} f(x) d x .
$$

Then

$$
\frac{1}{2 \tilde{f}} \int_{0}^{2 \tilde{f}} \phi(y) d y \geq \frac{1}{b-a} \int_{a}^{b} \phi(f(x)) d x .
$$

Karlin and Studden (cf. [3, p. 412]) gave a more general inequality as follows:

Theorem 1.3. Let $f$ be a non-negative continuous concave function on $[a, b]$, not identically zero, $\tilde{f}$ be defined in (3) and let $\phi$ be a convex function on $[c, 2 \tilde{f}-c]$ where $c$ satisfies $0<c \leq f_{\min }$ (where $f_{\min }$ is the minimum of $f$ ) and $\tilde{f}$ is defined in (3). Then

$$
\frac{1}{2 \tilde{f}-2 c} \int_{c}^{2 \tilde{f}-c} \phi(y) d y \geq \frac{1}{b-a} \int_{a}^{b} \phi(f(x)) d x
$$

For $\phi(y)=y^{p}, p>1$, we can get from Theorem 1.3: 
Theorem 1.4. Let $f$ be continuous concave function such that $0<c \leq f_{\min }, \tilde{f}$ is defined in (3). If $p>1$, then

$$
\frac{1}{(2 \tilde{f}-2 c)(p+1)}\left((2 \tilde{f}-c)^{p+1}-c^{p+1}\right) \geq \frac{1}{b-a} \int_{a}^{b} f^{p}(x) d x .
$$

If $0<p<1$, then the reverse inequality holds in (6).

Definnition 1.5. A function $h:(a, b) \rightarrow \mathbb{R}$ is exponentially convex function if it is continuous and

$$
\sum_{i, j=1}^{n} \xi_{i} \xi_{j} h\left(x_{i}+x_{j}\right) \geq 0
$$

for all $n \in \mathbb{N}$ and all choices $\xi_{i} \in \mathbb{R}$ and $x_{i} \in(a, b), i=1, \ldots, n$ such that $x_{i}+x_{j} \in(a, b), 1 \leq i, j \leq n$.

The following proposition is given in [4]:

Proposition 1.6. Let $h:(a, b) \rightarrow \mathbb{R}$. The following propositions are equivalent.

(i) $h$ is exponentially convex.

(ii) $h$ is continuous and

$$
\sum_{i, j=1}^{n} \xi_{i} \xi_{j} h\left(\frac{x_{i}+x_{j}}{2}\right) \geq 0,
$$

for every $n \in \mathbb{N}$, every $\xi_{i} \in \mathbb{R}$ and every $x, x \in(a, b), 1 \leq i, j \leq n$.

Corollary 1.7. If $h$ is exponentially convex then

$$
\operatorname{det}\left[h\left(\frac{x_{i}+x_{j}}{2}\right)\right]_{i, j=1}^{n} \geq 0,
$$

for every $n \in \mathbb{N}$, and every $x_{i} \in(a, b), i=1, \ldots, n$. 
Corollary 1.8. If $h:(a, b) \rightarrow \mathbb{R}^{+}$is exponentially convex function then $h$ is a log-convex functions.

With the help of following useful Lemma we prove our results:

Lemma 1.9. Define the functions

$$
\varphi_{S}(x)=\left\{\begin{array}{lc}
\frac{x^{s}}{s(s-1)}, & s \neq 0,1 ; \\
-\log x, & s=0 ; \\
x \log x, & s=1 .
\end{array}\right.
$$

Then $\varphi_{S}^{\prime \prime}(x)=x^{s-2}$, that is, $\varphi_{S}(x)$ is convex for $x>0$.

In second section we prove positive semi-definiteness of matrices generated by differences deduced from Favard's inequalities (2) and (6). This implies a surprising property of exponential convexity of this differences which allows us to deduce Gram's, Lyapunov's and Dresher's types of inequalities for this differences. Our results are extensions of results for log-convexity given in [5].

\section{FAVARD'S INEQUALITY}

Theorem 2.1. Let $f$ be a positive continuous concave function on $[a, b]$, $\tilde{f}$ be defined in (3) and

$$
\Omega_{s}(f):=\left\{\begin{array}{rr}
\frac{1}{s(s-1)}\left[\frac{2^{s}}{x+1}\left(\frac{1}{b-a} \int_{a}^{b} f(x) d x\right)^{s}-\frac{1}{b-a} \int_{a}^{b} f^{s}(x) d x\right], & s \neq 0,1, \\
1-\log 2-\log \tilde{f}+\frac{1}{b-a} \int_{a}^{b} \log f(x) d x, & s=0, \\
\log 2 \tilde{f}+\tilde{f} \log \tilde{f}-\frac{1}{2} \tilde{f}-\frac{1}{b-a} \int_{a}^{b} f(x) \log f(x) d x, & s=1 .
\end{array}\right.
$$

Then the following statements are valid: 
(a) For every $n \in \mathbb{N}$ and $s_{1}, \ldots, s_{n} \in \mathbb{R}$, the matrix $\left[\frac{\Omega_{s_{i}+s_{j}}}{2}\right]_{i, j=1}^{n}$ is a positive semi-definite matrix. Particularly

$$
\operatorname{det}\left[\frac{\Omega_{s_{i}+s_{j}}}{2}\right]_{i, j=1}^{k} \geq 0
$$

for $k=1, \ldots, n$.

(b) The function $s \rightarrow \Omega_{s}$ is exponentially convex.

(c) The function $s \rightarrow \Omega_{s}$ is a log-convex on $\mathbb{R}$ and the following inequality holds for $-\infty<r<s<t<\infty$ :

$$
\Omega_{s}^{t-r} \leq \Omega_{r}^{t-s} \Omega_{t}^{s-r}
$$

Proof. (a) Consider the function

$$
\phi(x)=\sum_{i, j}^{k} u_{i} u_{j} \varphi_{s_{i j}}(x)
$$

for $k=1, \ldots, n, x>0, u_{i} \in \mathbb{R}, s_{i j} \in \mathbb{R}$, where $s_{i j}=\frac{s_{i}+s_{j}}{2}$ and $\varphi_{s_{i j}}$ is defined in (7). Set

$$
\phi(x)=\sum_{i, j}^{k} u_{i} u_{j} \varphi_{s_{i j}}(x)
$$

We have

$$
\begin{aligned}
\phi^{\prime \prime}(x) & =\sum_{i, j}^{k} u_{i} u_{j} x^{s_{i j}-2} \\
& =\left(\sum_{i}^{k} u_{i} x^{\frac{s_{i}-1}{2}}\right)^{2} \geq 0, \quad x \geq 0 .
\end{aligned}
$$

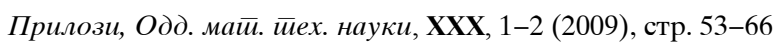


This shows that $\phi$ is a convex function for $x \geq 0$.

Using Theorem 1.2,

$$
\frac{1}{2 \tilde{f}} \int_{0}^{2 \tilde{f}} \phi(y) d y \geq \frac{1}{b-a} \int_{a}^{b} \phi(f(x)) d x,
$$

we have

$$
\begin{aligned}
& \frac{1}{2 \tilde{f}} \int_{0}^{2 \tilde{f}}\left(\sum_{i, j}^{k} u_{i} u_{j} \varphi_{s_{i, j}}(y)\right) d y \\
& \quad \geq \frac{1}{b-a} \int_{a}^{b}\left(\sum_{i, j}^{k} u_{i} u_{j} \varphi_{s_{i, j}}(f(x))\right) d x
\end{aligned}
$$

or equivalently

$$
\sum_{i, j}^{k} u_{i} u_{j} \Omega_{s_{i, j}} \geq 0
$$

From last inequality, it follows that the matrix $\left[\Omega_{\frac{s_{i}+s_{j}}{2}}\right]_{i, j=1}^{k}$ is a positive semi-definite matrix, that is, (9) is valid.

(b) Note that $\Omega_{s}$ is continuous for $s \in \mathbb{R}$ since

$$
\lim _{s \rightarrow 0} \Omega_{s}=\Omega_{0} \quad \text { and } \quad \lim _{s \rightarrow 1} \Omega_{s}=\Omega_{1}
$$

Then by using Proposition 1.6, we get exponentially convexity of the function $s \rightarrow \Omega_{s}$.

(c) A simple consequence of Corollary 1.8 is that $\Omega_{s}$ is log-convex, then by definnition

$$
\log \Omega_{s}^{t-r} \leq \log \Omega_{r}^{t-s}+\log \Omega_{t}^{S-r}
$$

which is equivalent to (10). 
Remark 2.2. Theorem 2.1 (c) was proved in [5].

Remark 2.3. The following result was also proved in [5] as a consequence of Theorem 2.1 (c).

Let $f, \Omega_{s}(f)$ be defined in Theorem 2.1 and $t, s, u, v$ be real numbers such that $s \leq u, t \leq v, s \neq t, u \neq v$, we have

$$
\left(\frac{\Omega_{S}(f)}{\Omega_{S}(f)}\right)^{\frac{1}{t-s}} \leq\left(\frac{\Omega_{v}(f)}{\Omega_{u}(f)}\right)^{\frac{1}{v-u}}
$$

Namely, in (cf [6], p.2), we have the following result for convex function $f$ with $x_{1} \leq y_{1}, x_{2} \leq y_{2}, x_{1} \neq x_{2}, y_{1} \neq y_{2}$,

$$
\frac{f\left(x_{2}\right)-f\left(x_{1}\right)}{x_{2}-x_{1}} \leq \frac{f\left(y_{2}\right)-f\left(y_{1}\right)}{y_{2}-y_{1}} .
$$

Since by Theorem 2.1, $\Omega_{s}(f)$ is log-convex, we can set in (12): $f(x)=\log \Omega_{x}$ and $x_{1}=s, x_{2}=t, y_{1}=u, y_{2}=v$. We get

$$
\begin{gathered}
\frac{\log \Omega_{t}(f)-\log \Omega_{S}(f)}{t-s} \leq \frac{\log \Omega_{v}(f)-\log \Omega_{u}(f)}{v-u}, \\
\log \left(\frac{\Omega_{t}(f)}{\Omega_{s}(f)}\right)^{\frac{1}{t-s}} \leq \log \left(\frac{\Omega_{v}(f)}{\Omega_{u}(f)}\right)^{\frac{1}{v-u}},
\end{gathered}
$$

after applying exponential function, we get (11).

Theorem 2.4. Let $f$ be a continuous concave function on $[a, b]$ such that $0 \leq c \leq f_{\min }, \tilde{f}$ be defined in (3) and 


$$
\Lambda_{s}(f):=\left\{\begin{array}{r}
\frac{1}{s(s-1)}\left[\frac{(2 \tilde{f}-c)^{s+1}}{(2 \tilde{f}-2 c)(s+1)}-\frac{c^{s+1}}{(2 \tilde{f}-2 c)(s+1)}-\frac{1}{b-a} \int_{a}^{b} f^{s}(x) d x\right], \quad s \neq 0,1, \\
\frac{1}{2 \tilde{f}-2 c}[2 \tilde{f}+c \log c-2 c-(2 \tilde{f}-c) \log (2 \tilde{f}-c)] \\
+\frac{1}{b-a} \int_{a}^{b} \log f(x) d x, \quad s=0, \\
\frac{1}{2 \tilde{f}-2 c}\left[(2 \tilde{f}-c)^{2} \log (2 \tilde{f}-c)-2 \tilde{f}^{2}+2 c \tilde{f}-c^{2} \log c+2 c\right] \\
-\frac{1}{b-a} \int_{a}^{b} f(x) \log f(x) d x, \quad s=1 .
\end{array}\right.
$$

Then the following statements are valid:

(a) For every $n \in \mathbb{N}$ and $s_{1}, \ldots, s_{n} \in \mathbb{R}$, the matrix $\left[\frac{\Lambda_{s_{i}+s_{j}}}{2}\right]_{i, j=1}^{n}$ is a positive semi-definite matrix. Particularly

$$
\operatorname{det}\left[\frac{\Lambda_{s_{i}+s_{j}}}{2}\right]_{i, j=1}^{k} \geq 0
$$

for $k=1, \ldots, n$.

(b) The function $s \rightarrow \Lambda_{s}$, is exponentially convex.

(c) The function $s \rightarrow \Lambda_{s}$, is a log-convex on $\mathbb{R}$ and the following inequality holds for $-\infty<r<s<t<\infty$ :

$$
\Lambda_{s}^{t-r} \leq \Lambda_{r}^{t-s} \Lambda_{t}^{s-r} .
$$

Proof. As in the proof of Theorem 2.1, we use Theorem 1.4 instead of Theorem 1.2.

Remark 2.5. Theorem 2.4 (c) was proved in [5]. 
Remark 2.6. The following result was also proved in [5] as a consequence of Theorem 2.4 (c).

Let $f, \Lambda_{s}(f)$ be defined in Theorem 2.4 and $t, s, u, v$ be real numbers such that $s \leq u, t \leq v, s \neq t, u \neq v$, we have

$$
\left(\frac{\Lambda_{t}(f)}{\Lambda_{s}(f)}\right)^{\frac{1}{t-s}} \leq\left(\frac{\Lambda_{v}(f)}{\Lambda u(f)}\right)^{\frac{1}{v-u}} .
$$

\section{WEIGHTED FAVARD'S INEQUALITY}

The weighted version of Favard's inequality was obtained by L. Maligranda, J. E. Pečarić and L. E. Persson in [6].

\section{Theorem 3.1.}

(1) Let $f$ be a positive increasing concave function on $[a, b]$. Assume that $\phi$ is a convex function on $[0, \infty)$, where

$$
\tilde{f}_{i}=\frac{(b-a) \int_{a}^{b} f(t) w(t) d t}{2 \int_{a}^{b}(t-a) w(t) d t} .
$$

Then

$$
\frac{1}{b-a} \int_{a}^{b} \phi(f(t)) w(t) d t \leq \int_{0}^{1} \phi\left(2 r \tilde{f}_{i}\right) w[a(1-r)+b r] d r .
$$

If $f$ is an increasing convex function on $[a, b]$ and $f(a)=0$, then the reverse inequality in (18) holds.

(2) Let $f$ be a positive decreasing concave function on $[a, b]$. Assume that $\phi$ is a convex function on $[0, \infty)$, where

$$
\tilde{f}_{d}=\frac{(b-a) \int_{a}^{b} f(t) w(t) d t}{2 \int_{a}^{b}(b-t) w(t) d t} .
$$


Then

$$
\frac{1}{b-a} \int_{a}^{b} \phi(f(t)) w(t) d t \leq \int_{0}^{1} \phi\left(2 r \tilde{f}_{d}\right) w[a r+b(1-r)] d r .
$$

If $f$ is a decreasing convex function on $[a, b]$ and $f(b)=0$, then the reverse inequality in (20) holds.

\section{Theorem 3.2.}

(1) Let $f$ be a positive increasing concave function on $[a, b], \tilde{f}_{i}$ is defined in (17) and

$$
\Pi_{s}(f):=\int_{0}^{1} \varphi_{S}\left(2 r \tilde{f}_{i}\right) w[a(1-r)+b r] d r-\frac{1}{b-a} \int_{a}^{b} \varphi_{S}(f(t) w(t) d t .
$$

Then the following statements are valid:

(a) For every $n \in \mathbb{N}$ and $s_{1}, \ldots, s_{n} \in \mathbb{R}$, the matrix $\left[\frac{\Lambda_{s_{i}+s_{j}}}{2}\right]_{i, j=1}^{n}$ is a positive semi-definite matrix. Paticularly

$$
\operatorname{det}\left[\frac{\Pi_{s_{i}+s_{j}}}{2}\right]_{i, j=1}^{k} \geq 0
$$

for $k=1, \ldots, n$.

(b) The function $s \rightarrow \Pi_{s}$ is exponentially convex.

(c) The function $s \rightarrow \Pi_{s}$ is a log-convex on $\mathbb{R}$ and the following inequality holds for $-\infty<r<s<t<\infty$ :

$$
\Pi_{s}^{t-r} \leq \Pi_{r}^{t-s} \Pi_{t}^{s-r}
$$

(2) Let $f$ be an increasing convex function on $[a, b], f(a)=0$, $\tilde{\Pi}_{s}(f):=-\Pi_{s}(f)$. Then the following statements are valid: 
(a) For every $n \in \mathbb{N}$ and $s_{1}, \ldots, s_{n} \in \mathbb{R}$, the matrix $\left[\frac{\tilde{\Pi}_{s_{i}+s_{j}}}{2}\right]_{i, j=1}^{n}$ is a positive semi-definite matrix. Particularly

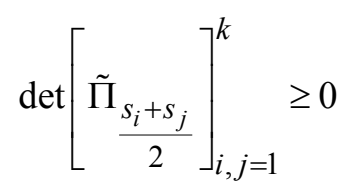

for $k=1, \ldots, n$.

(b) The function $s \rightarrow \tilde{\Pi}_{s}$ is exponentially convex.

(c) The function $s \rightarrow \tilde{\Pi}_{s}$ is a log-convex on $\mathbb{R}$ and the following inequality holds for $-\infty<r<s<t<\infty$ :

$$
\tilde{\Pi}_{s}^{t-r} \leq \tilde{\Pi}_{r}^{t-s} \tilde{\Pi}_{t}^{s-r}
$$

Proof. As in the proof of Theorem 2.1, we use Theorem 3.1(1) instead of Theorem 1.2.

Remark 3.3. Theorem 3.2 (c) was proved in [5].

Remark 3.4. The following result was also proved in [5] as a consequence of Theorem 3.2 (c).

(1) Let $f$ and $\Pi_{s}(f)$ be defined as in Theorem 3.2(1) and $t, s, u, v \geq 0$ be such that $s \leq u, t \leq v, s \neq t, u \neq v$. Then

$$
\left(\frac{\Pi_{t}(f)}{\Pi_{s}(f)}\right)^{\frac{1}{t-s}} \leq\left(\frac{\Pi_{v}(f)}{\Pi_{u}(f)}\right)^{\frac{1}{v-u}}
$$

(2) Let $f$ and $\tilde{\Pi}_{s}(f)$ be defined as in Theorem 3.2(2) and $t, s, u, v \geq 0$ such that $s \leq u, t \leq v, s \neq t, u \neq v$. Then

$$
\left(\frac{\tilde{\Pi}_{t}(f)}{\tilde{\Pi}_{s}(f)}\right)^{\frac{1}{t-s}} \leq\left(\frac{\tilde{\Pi}_{v}(f)}{\tilde{\Pi}_{u}(f)}\right)^{\frac{1}{v-u}}
$$




\section{Theorem 3.5.}

(1) Let $f$ be a positive decreasing concave function on $[a, b], \tilde{f}_{d}$ be defined as in (19) and

$$
\Gamma_{S}(f):=\int_{0}^{1} \varphi_{S}\left(2 r \tilde{f}_{d}\right) w[a r+b(1-r)] d r-\frac{1}{b-a} \int_{a}^{b} \varphi_{S}(f(t)) w(t) d t .
$$

Then the following statements are valid:

(a) For every $n \in \mathbb{N}$ and $s_{1}, \ldots, s_{n} \in \mathbb{R}$, the matrix $\left[\frac{\Gamma_{s_{i}+s_{j}}}{2}\right]_{i, j=1}^{n}$ is a positive semi-definite matrix. Particularly

$$
\operatorname{det}\left[\frac{\Gamma_{s_{i}+s_{j}}}{2}\right]_{i, j=1}^{k} \geq 0
$$

for $k=1, \ldots, n$.

(b) The function $s \rightarrow \Gamma_{s}$ is exponentially convex.

(c) The function $s \rightarrow \Gamma_{s}$ is a log-convex on $\mathbb{R}$ and the following inequality holds for $-\infty<r<s<t<\infty$ :

$$
\Gamma_{s}^{t-r} \leq \Gamma_{r}^{t-s} \Gamma_{t}^{S-r}
$$

(2) Let $f$ be a decreasing convex function on $[a, b], f(b)=0$, $\tilde{\Gamma}_{s}(f):=-\Gamma_{s}(f)$. Then the following statements are valid:

(a) For every $n \in \mathbb{N}$ and $s_{1}, \ldots, s_{n} \in \mathbb{R}$, the matrix $\left[\tilde{\Gamma}_{\frac{s_{i}+s_{j}}{2}}\right]_{i, j=1}^{n}$ is a positive semi-definite matrix. Paticularly

$$
\operatorname{det}\left[\frac{\tilde{\Gamma}_{s_{i}+s_{j}}}{2}\right]_{i, j=1}^{k} \geq 0
$$

for $k=1, \ldots, n$. 
(b) The function $s \rightarrow \tilde{\Gamma}_{s}$ is exponentially convex.

(c) The function $s \rightarrow \tilde{\Gamma}_{s}$ is a log-convex on $\mathbb{R}$ and the following inequality holds for $-\infty<r<s<t<\infty$ :

$$
\tilde{\Gamma}_{s}^{t-r} \leq \tilde{\Gamma}_{r}^{t-s} \tilde{\Gamma}_{t}^{s-r}
$$

Proof. As in the proof of Theorem 2.1, we use Theorem 3.1(2) instead of Theorem 1.2.

Remark 3.6. Theorem 3.5 (c) was proved in [5].

Remark 3.7. The following result was also proved in [5] as a consequence of Theorem 3.5 (c).

(1) Let $f$ and $\Gamma_{s}(f)$ be defined as in Theorem 3.5(1) and $t, s, u, v \geq 0$ be such that $s \leq u, t \leq v, s \neq t, u \neq v$. Then

$$
\left(\frac{\Gamma_{t}(f)}{\Gamma_{S}(f)}\right)^{\frac{1}{t-s}} \leq\left(\frac{\Gamma_{v}(f)}{\Gamma_{u}(f)}\right)^{\frac{1}{v-u}}
$$

(2) Let $f$ and e $\widehat{\Gamma}_{s}(f)$ be defined as in Theorem 3.5(2) and $t ; s ; u ; v \geq 0$ such that $s \leq u, t \leq v, s \neq t, u \neq v$. Then

$$
\left(\frac{\tilde{\Gamma}_{t}(f)}{\tilde{\Gamma}_{s}(f)}\right)^{\frac{1}{t-s}} \leq\left(\frac{\tilde{\Gamma}_{v}(f)}{\tilde{\Gamma}_{u}(f)}\right)^{\frac{1}{v-u}} .
$$

\section{REFERENCES}

[1] G. H. Hardy, J. E. Littlewood, G. Pólya: Inequalities, Cambridge University Press, Cambridge, 1978.

[2] D. S. Mitrinović: Analytic Inequalities, Springer-Verlag, Berlin, 1970.

[3] S. Karlin, W. J. Studden: Tchebycheff Systems, with Applications in Analysis and Statistics, Interscience, New York, 1966.

[4] Matloob Anwar, J. Jakšetić, J. Pečarić and Atiq ur Rehman: Exponential convexity, positive semi-definite matrices and fundamental inequalities, submitted for publication J. Math. Inequal. to appear.

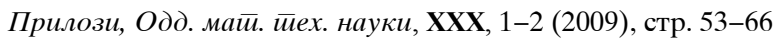


[5] Naveed Latif, J. Pečarić and I. Perić: Some new results related to Favard's inequality, J. Inequal. Appl, volume 2009, Article ID 128486, 14 pages, doi: $10.115 / 2009 / 128486$

[6] J. Pečarić, F. Proschan and Y. L. Tong: Convex functions, Partial Orderings and Statistical Applications, Academic Press, New York, 1992.

[7] T. Popoviciu. Sur quelques inégalités enter les fonctions convexes (I-III). C. $R$ Inst. Sc. Roumaine 2, 449-454, 454-458, and 3, 396-402 (1939).

Р е $з$ и м е

\section{ЕКСПОНЕНЦИЈАЛНА КОНВЕКСНОСТ НА FAVARD-ОВОТО НЕРАВЕНСТВО И СРОДНИ РЕЗУЛТАТИ}

Во оваа статија ние докажуваме позитивна полудефинираност на матрици генерирани од разлики изведени од нетежински и тежински Favard-ови неравенства. Ова имплицира едно интересно својство на експоненцијалната конвексност на овие разлики, кои ни даваат можност да ги изведеме Gram-овите, Lyapunov-ите и Dresher-овите типови неравенства за овие разлики.

Клучни зборови: Favard-ово неравенство; тежинско Favard-ово неравенство; позитивна полудефинитна матрица; експоненцијална конвексност; log-конвексност

\section{Address:}

Naveed Latif

Abdus Salam School of Mathematical Sciences, GC University,

Lahore, Pakistan

E-mail: sincerehumtum@yahoo.com

Josip Pečarić

Abdus Salam School of Mathematical Sciences, GC University,

Lahore, Pakistan

Faculty of Textile Technology, University of Zagreb,

Zagreb, Croatia

E-mail: pecaric@mahazu.hazu.hr

\section{Ivan Perić}

Faculty of Food Technology and Biotechnology, University of Zagreb,

Zagreb, Croatia

E-mail: iperic@pbf.hr

Received: 22. X 2009

Accepted: 1. XII 2009 